\title{
Hans Staden oder das Leben an den Rändern einer sich globalisierenden Welt
}

Von zahlreichen Mühen und Gefahren, aber auch mehreren Schiffbrüchen konnte auch der zwischen 1525 und 1528 geborene Hans Staden aus Homberg bei Kassel berichten. Seine Warhaftige Historia und Beschreibung eyner Landtschafft der Wilden, Nacketen, Grimmigen Menschfresser Leuthen war im Jahre 1557 erschienen, wurde in der Folge in zahlreiche Sprachen übersetzt und gilt bis heute als einer der Grundlagentexte zur Geschichte Brasiliens und des europäisch-überseeischen Kulturkontakts. ${ }^{1}$ Wir haben es also nicht mit einem marginalen Text, sondern mit einem tatsächlichen Klassiker des 16. Jahrhunderts zu tun.

Dieser Reisebericht, der eine lange und durchaus spannende Rezeptionsgeschichte aufzuweisen hat, innerhalb derer sich eine prä-anthropologische, eine anthropologische, eine literarische und eine literaturkritische Lesart voneinander unterscheiden lassen, ${ }^{2}$ bietet eine manche Elemente des pikaresken Romans, der sich zeitgenössisch auch in Deutschland zu verbreiten begann, aufnehmende, zugleich aber gerade die indigenen Kulturen der Tupi-Indianer beschreibende Struktur an, die ihren großen Erfolg den eher 'abenteuerlich' anmutenden Passagen verdankt. Es gibt keinen Zweifel angesichts des Erfolg dieses Bandes: Das Werk lässt sich als „Bestseller“ des 16. Jahrhunderts bezeichnen. ${ }^{3}$

Nach einer ersten, zwischen 1547 und 1549 unter portugiesischer Führung nach Brasilien unternommenen Reise geriet der hessische Armbrustschütze und Kanonier 1554, nach dem Schiffbruch seines spanischen Schiffes und einem waghalsigen Rettungsversuch, bei seinem zweiten Aufenthalt an der heute brasilianischen Küste für etwa neun Monate in die Gefangenschaft der Tupinambás und musste zusehen, wie diese einige seiner Mitgefangenen verspeisten. Hans Staden selbst überlebte - sonst wäre es auch nie zu diesem Buch gekommen. Doch stellen wir die Erklärungen für das Überleben des Homberger Söldners noch ein wenig zurück.

\footnotetext{
1 Vgl. hierzu die faksimilierte Ausgabe von Staden, Hans: Warhaftige Historia und Beschreibung eyner Landtschafft der Wilden, Nacketen, Grimmigen Menschfresser Leuthen [...]. Marpurg. Faksimile-Ausgabe. Frankfurt am Main 1925; ich benutze im folgenden die leichter zugängliche Edition von Staden, Hans: Brasilien 1547-1555. Mit 62 Abbildungen und 1 Karte. Herausgegeben und eingeleitet von Gustav Faber. Aus dem Frühneuhochdeutschen übertragen von Ulrich Schlemmer. Stuttgart: Edition Erdmann im K. Thienemanns Verlag 1982.

2 So Münzel, Mark: Vier Lesarten eines Buches: Zur Rezeption von Hans Stadens 'Warhaftige Historia'. In: Martius-Staden-Jahrbuch (Sao Paulo) 53 (2006), pp. 9-22.

3 Ebda., S. 9.

Ә Open Access. (c) 2020 Ottmar Ette, publiziert von De Gruyter. (cc))BY-NC-ND Dieses Werk ist lizenziert unter der Creative Commons Attribution-NonCommericial-NoDerivatives 4.0 Lizenz.

https://doi.org/10.1515/9783110650686-013
} 


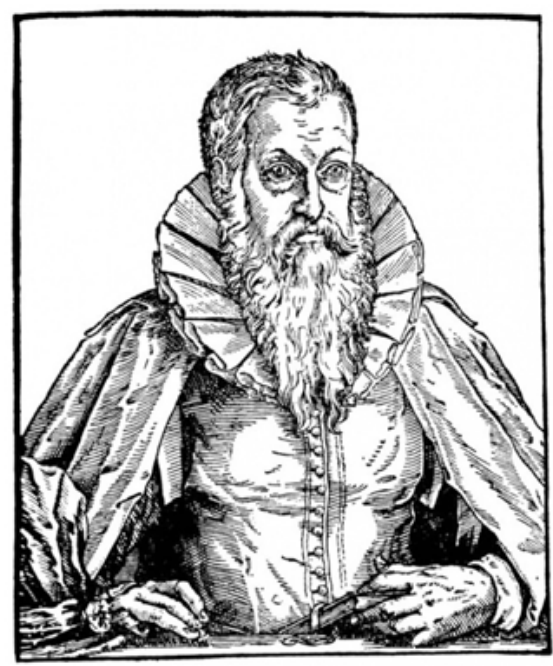

Abb. 42: Hans Staden (Homberg, um 1525 - Wolfhagen?, 1576).

Das Verspeisen des gekochten Fleisches eines zuvor gebratenen Christen brannte sich in Hans Stadens Schilderung des dafür veranstalteten Gelages ebenso stark in das Gedächtnis seiner Erzählerfigur ein wie die ergänzenden Bemerkungen, dass man „das Fleisch des anderen, des Jeronimo“, noch in jener Hütte aufbewahrte, „in der auch ich lebte“, und „fast drei Wochen lang in einem Korb über dem Feuer ${ }^{\star 4}$ konservierte. Gewiss sind dies Bilder, die keiner so leicht mehr vergisst. Erfahrung und Inszenierung von Alterität gehen in der Warhaftigen Historia freilich Hand in Hand.

Der Lebenslauf Hans Stadens, der in seinem bewegten Leben viermal den Atlantik überquerte, ist trotz der Aufsehen erregenden Schilderungen des von ihm Erlebten durchaus charakteristisch für jene zahlreichen Europäer, die - um das Jahr 1600 geht man von ungefähr 200000 Menschen aus Europa aus, die sich innerhalb des iberischen Kolonialreiches weltweit niedergelassen hatten ${ }^{5}$ - unterschiedlichste Posten innerhalb der Kolonialverwaltung einnahmen oder sich wie Staden als Söldner anwerben ließen. Hans Staden ist ein typischer Vertreter dieser großen Gruppe und zugleich das lebendige Beispiel dafür, dass in jeglicher Hinsicht die Globalisierung durch die Fahrt des Genuesen Columbus ein die spanische Welt weit übersteigendes Faktum war. Vom Beginn der ersten Phase beschleunigter Globalisierung an ist dieser Beschleunigungspozess ein gesamteuropäischer. Und dies

4 Staden, Hans: Brasilien, S. 186.

$5 \mathrm{Zu}$ diesen Schätzungen von Pierre Chaunu vgl. Gruzinski, Serge: Les Quatre Parties du monde, S. 251. 
schließt die Anwerbung ausländischer Soldaten, aber auch Arbeitskräfte, Schreiber, Matrosen und auch fremdländischer Marketenderinnen oder Packerinnen mit ein.

Die enorme Zahl an Menschen nicht nur aus Portugal, Spanien oder Italien, sondern aus vielen anderen Ländern Europas, die über transkontinentale Erfahrungen verfügten und - anders als Hans Staden - oftmals nicht mehr in ihre Heimat zurückkehrten, wird auch in der Warhaftigen Historia erkennbar, tauchen dort doch nicht nur portugiesische oder spanische Adelantados, Kapitäne oder einfache Beamte, sondern auch Landsleute wie Heliodorus Hessus, der es zum Faktor und Aufseher einer Zuckermühle brachte, oder Peter Roesel auf, der die Faktorei des ursprünglich aus Aachen stammenden Amsterdamer Bankiers und Kaufmanns Schetz leitete, der von São Vicente aus eine eigene Schiffsverbindung nach Antwerpen unterhielt. ${ }^{6}$ Die transatlantischen Bewegungen brachten eine bunte europäische Bevölkerung zusammen.

Wir dürfen also nicht von einer Globalisierungsphase ausgehen, die sich allein zwischen Spanien und Portugal ganz homogen abgespielt hätte. Beides waren zwar die Führungsmächte dieser Globalisierungsphase, aber deren Ausstrahlung und Auswirkungen reichten weit über die Iberische Halbinsel hinaus. Diese aus vielen Ländern stammenden Europäer bewegen sich bereits um die Mitte des 16. Jahrhunderts - also in einer Phase fortgesetzter, sich konsolidierender Globalisierung - in einem globalisierten Horizont, innerhalb dessen die noch für lange Zeit mit hohen Risiken behafteten Schiffsverbindungen dafür sorgen, dass Schiffbrüche oder die Angriffe von Piraten, Korsaren und Freibeutern im Bewusstsein der Zeitgenossen an der Tagesordnung sind. Diese waren ein geradezu 'natürliches' Phänomen des Mittelmeeres - nicht umsonst haben wir gesehen, dass selbst ein Columbus in seinen jungen Jahren ein recht erfolgreicher Korsar war -, so dass es alles andere als verwundert, wenn wir die Korsaren und Freibeuter nun auch die Weltbühne betreten sehen. Sie sollten sogar ein wahres Reich, einen Herrschaftsraum errichten, der über lange Zeit ein recht stabiles Machtgebilde ausgehend von der Karibik darstellte. In der ersten Phase beschleunigter Globalisierung wurden selbstverständlich auch die Freibeuter globalisiert.

Aber auch die Auseinandersetzungen und Kriege mit den zum damaligen Zeitpunkt zahlenmäßig noch weit überlegenen Tupi-Indianern führten zu Verlusten unter den europäischen Eindringlingen, weist doch auch Hans Stadens Bericht auf mehrfache Begegnungen mit anderen gefangenen 'Christen' - wie Staden sie bevorzugt nennt - sowie mit regelmäßig auftauchenden Franzosen

6 Vgl. hierzu Faber, Gustav: Vorwort. In: Staden, Hans: Brasilien, S. 43. 
hin, die sich mit den Indianern verbündeten, um sich im portugiesischen Kolonialreich festzusetzen. Es gab also durchaus unterschiedliche Kolonialinteressen der Europäer. Dies war von einem Hans Staden aus Homberg freilich nicht zu befürchten.

Rasch wurden von Anfang an auf dem gesamten amerikanischen Kontinent verschiedene indianische Völker und Gemeinschaften in die feindlichen Auseinandersetzungen zwischen miteinander rivalisierenden europäischen Nationen hineingezogen. So ergab sich eine Gemengelage an kriegerischen Aktivitäten, die man aus größerer historischer Distanz leicht einmal übersieht und als Randphänomen abtut. Für Hans Staden aber war dies täglich Brot seiner Aktivitäten.

Hans Staden lebte damit auch jeden Tag mit der Gefahr, vom Erdboden zu verschwinden - sein Beruf als Söldner war zweifellos mit vielerlei Risiken besetzt. Die Frage nach dem eigenen Überleben war daher gerade für zwar gebildete, aber gesellschaftlich nicht privilegierte Leute vom Schlage eines Hans Staden schon lange vor der Gefangennahme durch die Tupinambá allgegenwärtig. Keine Abkunft, keine Herkunft, keine Zukunft schützte ihn.

Doch die Gefangennahme durch die feindlichen Indianer - eine Art Schiffbruch 'zwischen' den wenigen Zentren europäischer Macht an der Ostküste Brasiliens - lässt den Homberger Söldner dann aber zu einem wahren Überlebenskünstler werden, der es wahrlich verstand, sein Überlebenswissen zu kultivieren und für sich selbst einzusetzen. In seinem späteren Bericht wurde der in den Diensten Portugals wie Spaniens arbeitende hessische Söldner nicht müde, gerade klimatische, aber auch viele andere Phänomene als Zeichen Gottes zu deuten und sein eigenes Überleben auf die Hilfe Gottes zurückzuführen. Auch er bevölkert wie Columbus die Welt mit göttlichen Zeichen und Symbolen, die ihn vor einem schnellen Tode retten. Und doch bietet der Text seiner Historia zugleich Anhaltspunkte dafür, warum gerade Hans Staden seine gefährliche Gefangenschaft überleben konnte.

Denn zweifellos war Staden auch ein ausgezeichneter Beobachter all jener Zeichen, die ihm vieles über die Lebensgewohnheiten, die Vorstellungen und Denkweisen der Indianer verrieten. Im Grunde war er ein Völkerkundler avant la lettre, ein wenig auch hier dem Columbus ähnlich, aber mit einem sichereren Gespür für die Realität. Zog der narrative erste Teil seines Bestsellers auch die meiste Aufmerksamkeit auf sich, so verfügt der zweite, diskursiv angelegte Teil über Qualitäten, die insbesondere im 20. Jahrhundert vorzugsweise ins Zentrum der (disziplinären) Auseinandersetzungen um Hans Staden rückten. Sehen wir uns dies einmal näher an.

Die beinahe wissenschaftlichen Qualitäten der diskursiven Teile dieses so erfolgreichen Reiseberichts sind verblüffend. Dabei scheint mir eine Deutung gerade 
dieses Teils im Sinne eines prä-anthropologischen Wissenschaftsprogramms ${ }^{7}$ ebenso zutreffend zu sein wie die Überzeugung, dass „die detaillierte Beschreibung ethnographischer Einzelheiten so genau mit heutiger wissenschaftlicher Kenntnis der Kultur der damaligen Tupinambá“ übereinstimmt, dass man sie getrost einem „Augenzeugen“ zuordnen könne. ${ }^{8}$ Im Übrigen zeigten auch die Holzschnitte, die selbstverständlich innerhalb des historischen, wissenschaftlichen und künstlerischen Horizonts ihrer Zeit verstanden werden müssten, „künstlerisch unbeholfen, aber mit großer ethnographischer Genauigkeit indianisches Leben, etwa Hütten oder Pflanzbau. “9 Es ist, als hätte Hans Staden seinen erzwungenen Aufenthalt in der ständigen Gefahr, verspeist zu werden, mit geradezu wissenschaftlichen Studien und seiner ethnographischen Neugier verkürzt und eine engagierte Feldforschung betrieben. Um sie dann später in Wort, aber auch Bild festzuhalten. Auch dies sind unzweifelhaft Fähigkeiten des literarischen Reiseberichts, wie wir ihn schon in dieser frühen Phase beschleunigter Globalisierung wahrnehmen können.

Aber wir sehen hier keinen Naturalisten des 18. Jahrhunderts am Werk. Die Darstellung der Fauna und vor allem der Flora an der brasilianischen Ostküste nahm in Stadens Reisebericht eine deutlich untergeordnete Rolle ein. Dafür interessierte er sich weit weniger, konnte dieser Bereich doch insgesamt auch wenig zu seiner eigenen Sicherheit und vor allem seinem Überleben beitragen.

Im Zentrum des zweiten Teiles von Hans Stadens Reisebericht stand ganz unverkennbar das Leben der Indianer, mit denen Staden auf für ihn unabsehbare Zeit zusammenleben musste. Staden erwies sich hier als ein genauer und präziser Beobachter und Beschreiber, dem auch vermeintliche Nebensächlichkeiten nicht entgingen. Mit großem Detailreichtum präsentierte der erfahrene Soldat die Konstruktionsweise der Wohnungen der Tupinambá, ihre Art, Feuer zu machen oder zu schlafen, ihre Geschicklichkeit bei der Jagd, beim Umgang mit ihren Waffen oder beim Anbau von Nutzpflanzen, die für die Gemeinschaft wichtig waren. Doch ging er nicht nur auf Werkzeuge oder Kochgewohnheiten, auf Trinksitten oder Schmuck ein, sondern fragte auch danach, „was bei ihnen Recht und Ordnung heißt“", ${ }^{10}$ welches ihre Glaubensvorstellungen sind, ${ }^{11}$ welchen Ehrbegriff sie pflegen ${ }^{12}$ oder wie Männer und Frauen zusammenleben. Er

\footnotetext{
7 Münzel, Mark: Vier Lesarten eines Buches, S. $10 \mathrm{f}$.

8 Ebda., S. 19.

9 Ebda.

10 Staden, Hans: Brasilien, S. 230.

11 Ebda., S. 242.

12 Ebda., S. 241.
} 


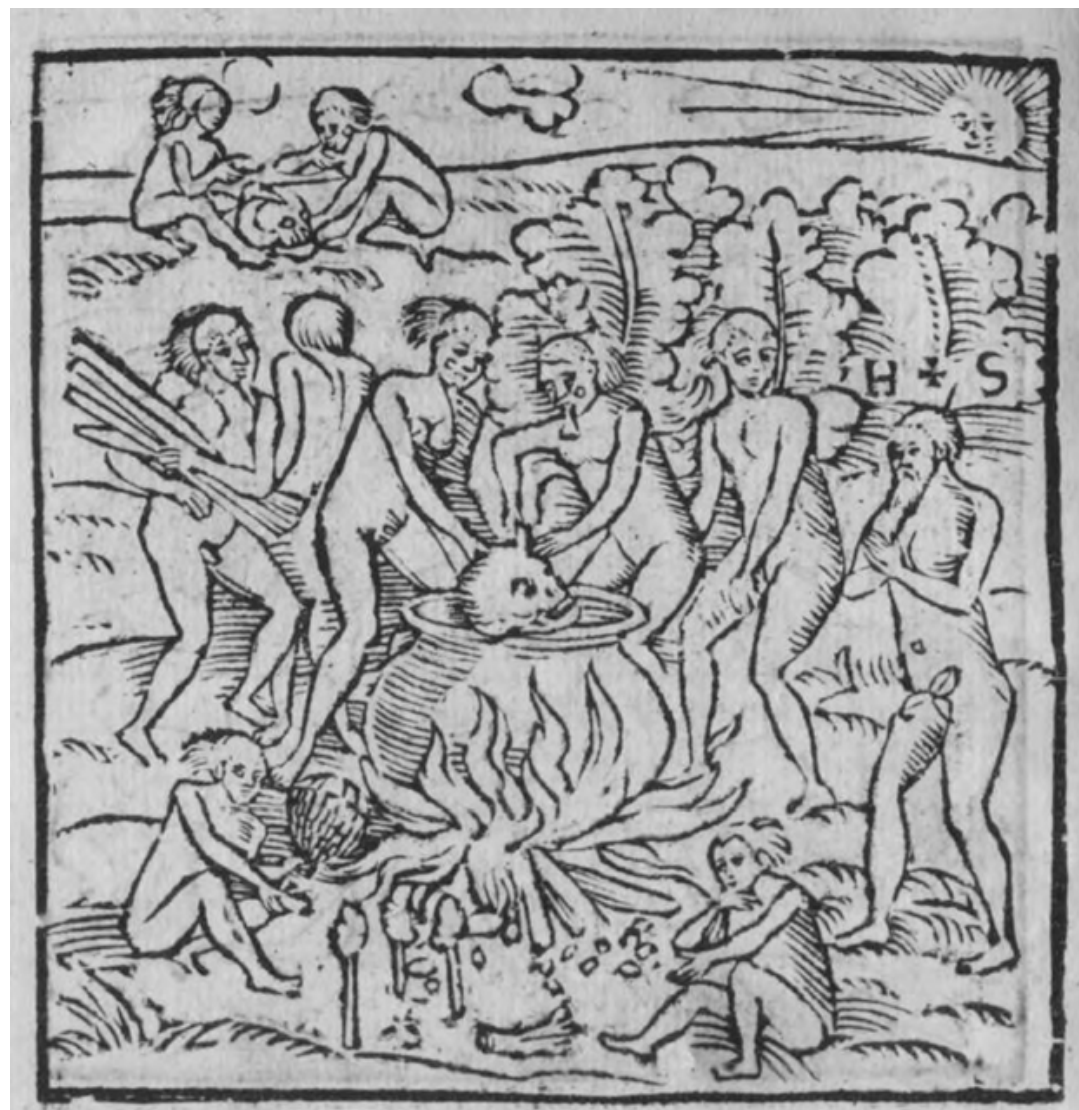

tSurien/effen ble fungen. Wann das alles gefdyeben ift/fe gebet dain cyn yeser wiberumb beym / onb nemen je ebeyl init fid. Dex ienige fo diefen getódtet bat/gibt fich nodb eye

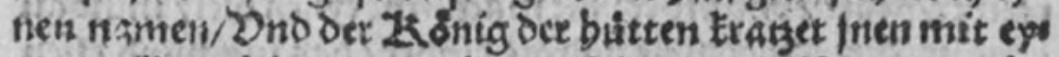

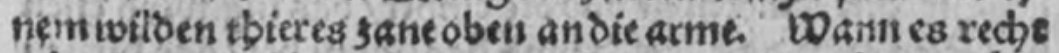
gebeylet ift/fo fibet mandie mafen/ $\partial_{a s}$ ift die ebre darfür.

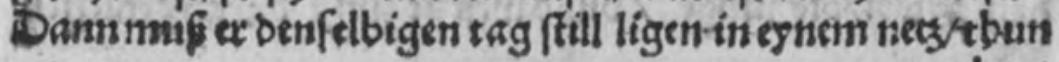
rome

Abb. 43: Kannibalismus in Brasilien gemäß der Beschreibung des Hans Staden. 
untersuchte damit vor allem ihre Gesellschaft als Gemeinschaft, die inneren Regeln ihres Zusammenlebens und die Grenzen einer Konvivenz, von der er selbst auch profitierte. Gerade diesen unterschiedlichen Aspekten gemeinschaftlichen Zusammenlebens schenkte er seine ungeteilte Aufmerksamkeit.

So heißt es etwa unter der Überschrift 'Wieviele Frauen ein Mann hat und wie er sich zu ihnen verhält':

Der größte Teil der Männer hat nur eine Frau, einige auch mehr. Manche Häuptlinge jedoch haben dreizehn bis vierzehn Frauen. Der Häuptling Abatí-poçanga, dem ich zum Schluß geschenkt wurde und von dem mich die Franzosen freikauften, hatte viele Frauen. Seine erste Frau hatte unter ihnen das höchste Ansehen. Jede hatte ihren eigenen Platz in der Hütte mit ihrem eigenen Feuer und ihren eigenen Maniokpflanzen. Mit welcher er gerade zusammen war, an deren Platz hielt er sich auf, und sie gab ihm zu essen. So ging das reihum. Die Knaben unter ihren Kindern ziehen auf die Jagd. Alles was sie erbeuten, bringen sie ihrer Mutter, die es kocht und mit den anderen Frauen teilt. Die Frauen vertragen sich recht gut untereinander. Es ist auch üblich, daß ein Mann seine Frau, wenn er ihrer überdrüssig ist, einem anderen schenkt. Ebenso schenken sie einander oft ihre Töchter oder Schwestern. ${ }^{13}$

Die vollständige Wiedergabe dieses Kapitels zeigt, wie detailliert die Beziehungen zwischen den Geschlechtern dargestellt und wie aufmerksam dabei die Formen des Zusammenlebens präsentiert werden. Hans Staden erweist sich hier als ein Beobachter, der um ein Höchstmaß an analytischer Objektivität bemüht ist und alles festhält, was von Bedeutung sein könnte. Staden entfaltet dergestalt ein recht komplexes Wissen über das Leben der Indianer, wobei sich seine Beobachtungen insbesondere auf ihre spezifischen Lebensformen und die Spielregeln des Zusammenlebens erstrecken. Aber wozu diente dieses Wissen? Und war es für ihn selbst von irgendeinem Nutzen?

Das von ihm zusammengestellte Lebenswissen war - so darf vermutet werden - für ihn ein ÜberLebenswissen, ${ }^{14}$ das es ihm ermöglichte, als Gefangener seinen Platz innerhalb der indianischen Gemeinschaft zu verstehen und sich an die nunmehr auch für ihn als Gefangenen geltenden Spielregeln zu halten. Dies bedeutete, dass er seinen Ort innerhalb der indianischen Gemeinschaft besser zu verstehen lernte und begriff, wo für ihn nicht sichtbare Gefahren, aber auch die Chancen seines Zusammenlebens lagen.

Zahlreich sind die Passagen des Reiseberichts, in denen von Interaktionen zwischen der Ich-Figur und den Indianern - seien sie sprachlicher oder nichtsprachlicher Art - die Rede ist. Staden untersuchte genau die Bedingungen und

13 Ebda., S. 238.

14 Vgl. hierzu Ette, Ottmar: ÜberLebenswissen. Die Aufgabe der Philologie. Berlin: Kulturverlag Kadmos 2004. 
Kontexte jeglicher Kommunikation innerhalb der Gemeinschaft. So entsteht ein tiefes Verständnis für die Formen des Zusammenlebens, innerhalb derer auch die Glaubensüberzeugungen der Ich-Figur selbst relevant werden und von den Indianern - etwa zur Beendigung eines ihre Pflanzungen gefährdenden Dauerregens - in ihrem Sinne funktionalisiert werden: Sie fordern Staden auf, seinen Gott um Hilfe anzuflehen. Und diese Aufforderung ist ein Faktum von ungeheurer Tragweite: Hans Staden wird in die Kommunikationsstruktur der indianischen Gemeinschaft miteinbezogen.

Damit kommt es zu einer wichtigen Wandlung. Was für die Ich-Figur ein Zeichen göttlichen Beistands ist, wird - als Bittgebet des Europäers an die Adresse seines Gottes - zu einem Element innerhalb der Ökonomie des Zusammenlebens innerhalb der indigenen Gemeinschaft. Als Antwort auf die oft und bisweilen misstrauisch gestellte Frage, warum unter den zahlreichen Europäern gerade Hans Staden nicht verspeist wurde, böte sich daher an, dieses ZusammenLebensWissen als ein Lebenswissen zu deuten, das der Ich-Figur - und wohl auch dem Autor selbst - das Überleben innerhalb einer anthropophagen Gemeinschaft ermöglichte, bevor sein Freikauf erneut eine Funktion innerhalb der indigenen Ökonomie der Tupinambá erfüllte. Mit anderen Worten: Hans Staden verstand die Bedingungen und Kontexte der Gesellschaft, innerhalb deren Spielregeln er leben musste, und dieses Verstehen, diese Hermeneutik, rettete ihm wohl das Leben.

Dass sich das von Hans Staden in literarischer Form gespeicherte und entfaltete ZusammenLebensWissen vorrangig auf eine individuelle Erfahrung und das Ziel eines Überlebens innerhalb einer zunächst gänzlich fremden und feindlichen Gemeinschaft, nicht aber auf generelle Formen und Möglichkeiten eines friedlichen Zusammenlebens zwischen Europäern und Indianern richtete, wird man dem Verfasser der Warhaftigen Historia wohl kaum vorwerfen können. Wie wäre dies auch möglich gewesen innerhalb europäischer Politiken, die wie selbstverständlich auf die eigene Stärke setzten und alles am $\mathrm{Zu}$ gewinn weiterer Territorien gerade auch auf dem amerikanischen Kontinent ausrichteten. Als angeheuerter Söldner in spanischen oder portugiesischen Diensten war er selbst Teil des Fehlers im System der kolonialen Ausbreitung Europas über die Welt, war selbst also ein Rädchen im kolonialen Expansionswerk Spaniens, Portugals und Europas. Genau in diesem Bereich siedelt sich auch die Signifikanz des von ihm entfalteten Verhältnisses von Reisen und Schreiben an.

So zeigt Hans Stadens Reisebericht auf faszinierende Weise, in welcher Form ein von seiner Zeit und ihren ästhetischen Formen und Normen geformter literarischer Text nicht nur für heutige Anthropologen überzeugende ethnographische Elemente, sondern auch ein Lebenswissen mit unterschiedlichsten Formen des ZusammenLebensWissens speichern kann, die bis heute von großer 
Relevanz und Faszinationskraft sind. Denn Hans Stadens Bericht liefert uns entscheidende Einsichten in die Lebensformen und Lebensnormen jener indigenen Gemeinschaften, die schon bald vor den europäischen Kolonisten kapitulieren mussten und weitestgehend verschwanden. Und selbst die aktuelle brasilianische Regierung Bolsonaro entblödet sich nicht, mit dem Ziel kurzfristiger Erzielung finanzieller Zugewinne langfristige Klimaschäden in Kauf zu nehmen und darüber hinaus das Risiko einzugehen, die wenigen verbliebenen indigenen Völker am Amazonas weitestgehend auszulöschen! An den Politiken der Nachfahren europäischer Kolonisten hat sich im Grunde wenig geändert.

In einem seiner berühmtesten Essais hat Michel de Montaigne unter dem Titel 'Des cannibales' seine erkenntnistheoretische Skepsis gegenüber einer vermeintlich vorurteilsfreien Wahrnehmung anderer Kulturen auf eine Formel gebracht, die in einem europäischen Text über Kannibalismus für sich selbst spricht: „J'ai peur que nous avons les yeux plus grands que le ventre, et plus de curiosité que nous n'avons de capacité. Nous embrassons tout, mais nous n'étraignons que du vent. “15

Wenn bei den Europäern aber die concupiscentia der Augen weit größer ist als das, was ihr Bauch - auch mit Blick auf die Berichte über Kannibalen - zu verdauen vermag, dann gelte es, mit äußerster Vorsicht zur Kenntnis zu nehmen, was gerade aus gebildeter (und daher stark mit Vorwissen ausgestatteter) europäischer Sicht über die Anthropophagen gesagt werde. Vielmehr hinterfragte der Franzose um 1580 meisterhaft die an Europa und den tradierten abendländischen Überzeugungen ausgerichteten und daher gleichsam 'natürlich' scheinenden Prämissen jeglicher Bewertung von Anthropophagie:

\footnotetext{
Or je trouve, pour revenir à mon propos, qu'il n'y a rien de barbare et de sauvage en cette nation, à ce qu'on m'en a rapporté, sinon que chacun appelle barbarie ce qui n'est pas de son usage; comme de vray, il semble que nous n'avons autre mire de la verité et de la raison que l'exemple et idées des opinions et usances du païs où nous sommes. Là est toujours la parfaicte religion, la parfaicte police, perfect et accomply usage de toutes choses. [...] car il me semble que ce que nous voyons par experience en ces nations là, surpasse non seulement toutes les peintures dequoy la poësie a embelly l'age doré et toutes ses inventions à feindre une heureuse condition d'hommes, mais encore la conception et le desir mesme de la philosophie. Ils n'ont peu imaginer une nayfveté si pure et simple, comme nous la voyons par experience; ny n'ont peu croire que nostre société se peut maintenir avec si peu d'artifice et de soudeure humaine. ${ }^{16}$
}

15 Montaigne: Essais. Chronologie et introduction par Alexandre Micha. Bd. I. Paris: GarnierFlammarion 1969, S. 251.

16 Ebda., Bd. I, S. 254 f. 


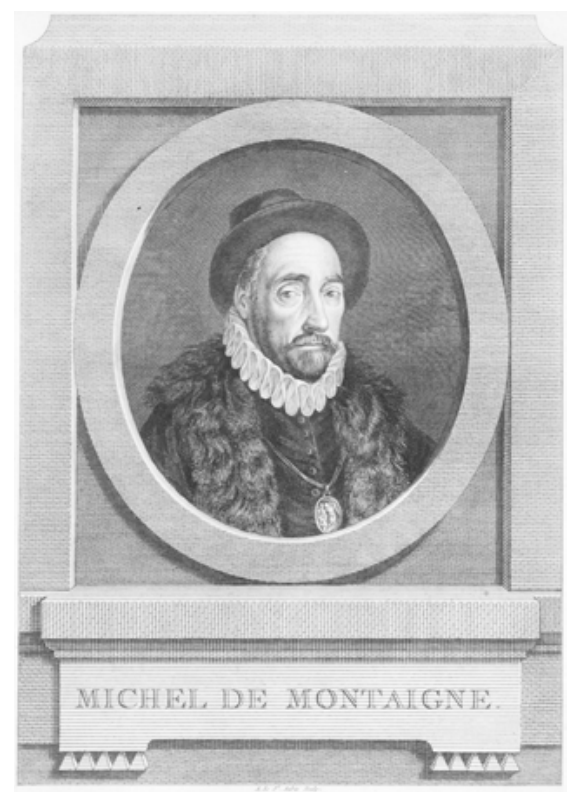

Abb. 44: Michel de Montaigne (Saint-Michelde-Montaigne in Périgord, 1533 - ebenda, 1592).

Michel de Montaigne prangert hier offen die beschränkten Wahrnehmungsgewohnheiten der Europäer und ihr rechthaberisches Verhalten an, sich selbst immer im Fluchtpunkt aller menschlichen Perfektion zu wissen, stets über die beste Ordnung, das beste Staatswesen zu verfügen. Und dies gerade auch in der Zeit Montaignes, wo all dies zu kollabieren drohte! Demgegenüber müsse dann weltweit alles hinter der eigenen, europäischen Perfektion zurückstehen und als defizitär gebrandmarkt werden.

Überdies entgeht Montaigne in dieser Passage der Todorov'schen Zwickmühle, ${ }^{17}$ entweder das Andere als Anderes anzuerkennen und dann jeweils positiv oder zumeist negativ zu bewerten oder aber eine Gleichheit anzuerkennen, die dann zum Auslöschen der Andersheit, der Alterität führt. Wir hatten die bis heute gültige Wirksamkeit dieser Zwickmühle ja bereits kennengelernt.

Der Autor der Essais führt hier vielmehr einen die eigene Kultur und deren Vorstellungen relativierenden, aber keineswegs eskamotierenden und verdrängenden Gedanken ein, der einen wichtigen Schritt zur Überwindung eines völlig selbstverständlich an europäischen Normen und Konventionen hängenden Projektionsapparates darstellt. Denn er wagt in seinem essai über

17 Vgl. Todorov, Tzvetan: Die Eroberung Amerikas, S. 56f. 
die Kannibalen vielmehr den Versuch, auf eine wahrnehmungs- und erkenntnistheoretisch begründete Weise ein kulturelle Grenzen überschreitendes Wissen vom Leben des Menschen zu formulieren, das in sich die Möglichkeiten eines Zusammenlebens in Differenz birgt.

Allen Europäern aber, die sich weit über der Stufe der Kannibalen glauben, schreibt er mit Blick auf die nicht enden wollenden Kriege und Bürgerkriege europäischer Machart mit deutlichen Worten ins Stammbuch:

Jugeans bien de leurs fautes, nous soyons si aveuglez aux nostres. Je pense qu'il y a plus de barbarie a manger un homme vivant qu'à le manger mort, à deschirer par tourmens et par geénes un corps encore plein de sentiment, le faire rostir par le menu, le faire mordre et meurtrir aux chiens et aux pourceaux (comme nous l'avons non seulement leu, mais veu de fresche memoire, non entre des ennemis anciens, mais entre des voisins et concitoyens, et, qui pis est, sous pretexte de pieté et de religion), que de le rostir et manger après qu'il est trespassé. [... .] Nous les pouvons donq bien appeller barbares, eu esgard aux regles de la raison, mais non pas eu esgard à nous, qui les surpassons en toute sorte de barbarie. $^{18}$

Montaigne blickt vorurteilslos auf seine eigene Zeit in Europa und führt all die Gräulichkeiten auf, die ihm noch in frischer Erinnerung sind, und fragt darauf, woher wir Europäer denn den Hochmut nähmen, so über die Indianer urteilen zu wollen. Denn würde man die absoluten Normen als Bezugspunkt wählen, so wären die indigenen Gruppen durchaus zu verurteilen, nicht aber dann, wenn wir sie mit dem konkreten Verhalten von Europäern in blutigen Bürgerkriegen, wie Montaigne sie erlebt hatte, in Vergleich setzen würden. Das Nicht-Reisen von Montaigne (der seinerseits sehr wohl Reisen innerhalb Europas unternahm) schließt keineswegs den Spielraum der Bewegung, die sich in seinen Texten zwischen Reisen und Schreiben eröffnet. Seine Essais greifen vielmehr auch auf Reiseberichte zurück und knüpfen an diese so lebendig und frei an, als wäre der Franzose auf allen Reisen geistig und intellektuell mit dabei gewesen.

Michel de Montaigne repräsentiert eine Selbstreflexion des europäischen Denkens, die sich immer wieder der eigenen Übergriffe, der eigenen beschränkten Wahrnehmungen und Exklusionen bewusst wird und diese anklagt. Schade nur, dass sich Europa immer nur zeitweise an derartige Traditionen erinnert! Denn wie sollten wir indigene Gruppen verurteilen, wo wir Abermillionen an Toten sinnlos in unseren Weltkriegen produzierten? Und wie könnten wir wohl eine eigene Überlegenheit begründen, wo wir die jahrhundertealten Fehler noch

18 Montaigne: Essais, Bd. I, S. $258 \mathrm{f}$. 
immer gerne begehen? Dies sind brennende Fragen, welche uns die Geschichte aufgibt. Doch es sind Fragen und Antworten, die wohl immer minoritär zu bleiben drohen, es sei denn, wir suchten gezielt nach jenen verschütteten Traditionen, die es in unserer Geschichte und vor allem in unseren Literaturen sehr wohl zu entdecken gilt. Denn dort findet sich ein Lebenswissen und ein Zusammenlebenswissen, vielleicht sogar ein Weiterlebenswissen gespeichert, die über die Jahrhunderte nichts von ihrer Aktualität für die heutige und künftige Konvivenz verloren haben. 